> La stimulation transcrânienne à courant continu est une technique de neuromodulation non invasive qui induit, selon la polarité de la stimulation, une augmentation ou une diminution de l'excitabilité corticale. Bien que son efficacité clinique ait été démontrée dans l'amélioration de déficits cognitifs survenant après un accident vasculaire cérébral, des travaux récents suggèrent également son utilité dans le traitement des troubles psychiatriques ainsi que sa valeur ajoutée dans la remédiation de processus psychopathogènes impliqués dans la chronification de ces troubles. Au vu de son faible coût, cette technique pourrait rapidement venir renouveler l'univers des soins de santé mentale. <

«Une pile de neuf volts, deux électrodes : la neurostimulation peut commencer $\gg$ titrait avec toquade le quotidien Le Monde en 2013, en vue d'évoquer les effets bénéfiques de la stimulation transcrânienne à courant continu (transcranial direct current stimulation: tDCS) pour soulager la douleur ou aider les patients à retrouver leurs capacités cognitives et motrices après un accident vasculaire cérébral. La tDCS consiste en l'induction, par l'intermédiaire de deux électrodes posées sur le scalp, d'un courant continu de faible intensité en vue de moduler l'activité neuronale des régions cérébrales localisées en dessous de ces électrodes (Figure 1). Le courant reste stable et continu durant la stimulation, générant ainsi un circuit (ou diode) dans lequel le courant circule de l'anode vers la cathode. Cette induction mène à une hyperexcitabilité des neurones des régions corticales situées sous l'anode et à une hypoexcitabilité de ceux des régions situées en regard de la cathode. En comparaison des autres méthodes de neuromodulation, la tDCS a pour avantage d'être simple, non invasive et peu coûteuse (Tableau 1). En outre, les effets secondaires sont relativement faibles et généralement limités à la manifestation transitoire de picotements ou de

\section{La stimulation transcrânienne à courant continu en psychiatrie}

Vers de nouvelles perspectives d'interventions

\author{
Alexandre Heeren ${ }^{1,2}$, Charlotte Coussement ${ }^{2,3}$, \\ Élisabeth Colon ${ }^{4,5}$
}

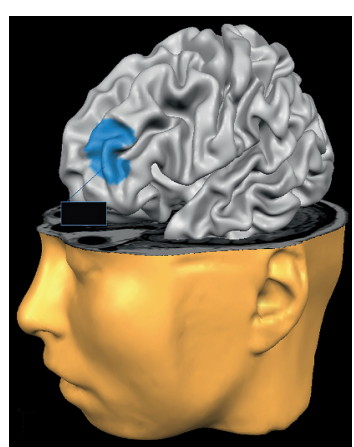

${ }^{1}$ Department of Psychology, 1232 William James Hall, Harvard university, 33 Kirkland street, Cambridge, MA 02138, États-Unis ;

${ }^{2}$ Institut de recherche en sciences psychologiques, université catholique de Louvain, Louvain-la-Neuve, Belgique ; ${ }^{3}$ Cellule de recherches et publications scientifiques (CRPS), hôpital psychiatrique du Beau Vallon, Namur, Belgique;

${ }^{4}$ Institut de neuroscience,

démangeaisons sous les électrodes de stimulation [1]. Au vu de ces qualités, l'application clinique de cette technique a rapidement suscité un réel enthousiasme. Ainsi, de nombreuses études ont-elles été conduites en vue d'évaluer l'efficacité clinique de la tDCS dans l'amélioration de déficits moteurs université catholique de Louvain, Bruxelles, Belgique;

${ }^{5}$ Center for Pain and the Brain, Department of Anesthesiology, Perioperative and Pain Medicine, Boston Children's Hospital, Harvard Medical School, Boston, MA, États-Unis

alexandreheeren@fas.harvard.edu ou langagiers survenant après un accident vasculaire cérébral, dans la réduction des acouphènes chroniques invalidants ou de la douleur chronique [2]. Au fil du temps, cet engouement s'est également généralisé aux troubles psychiatriques. En France, le coût annuel des troubles psychiatriques étant estimé à plus de 100 milliards d'euros [3], le développement d'outils thérapeutiques efficaces et à moindre coût constitue un défi majeur. Sa simplicité d'utilisation, son caractère non invasif, ainsi que son aspect extrêmement peu coûteux, ont rapidement fait de la tDCS un candidat d'espoir dans cette voie. Afin d'offrir un rapide panorama de ces avancées, l'objectif de cet article est de présenter une synthèse non-exhaustive de ces travaux. Le contexte d'apparition de la tDCS ainsi que son mode d'action seront d'abord brièvement présentés. Les études cliniques examinant son efficacité en psychiatrie seront ensuite exposées. Enfin, les perspectives actuelles de recherche-développement dans ce secteur seront discutées. 


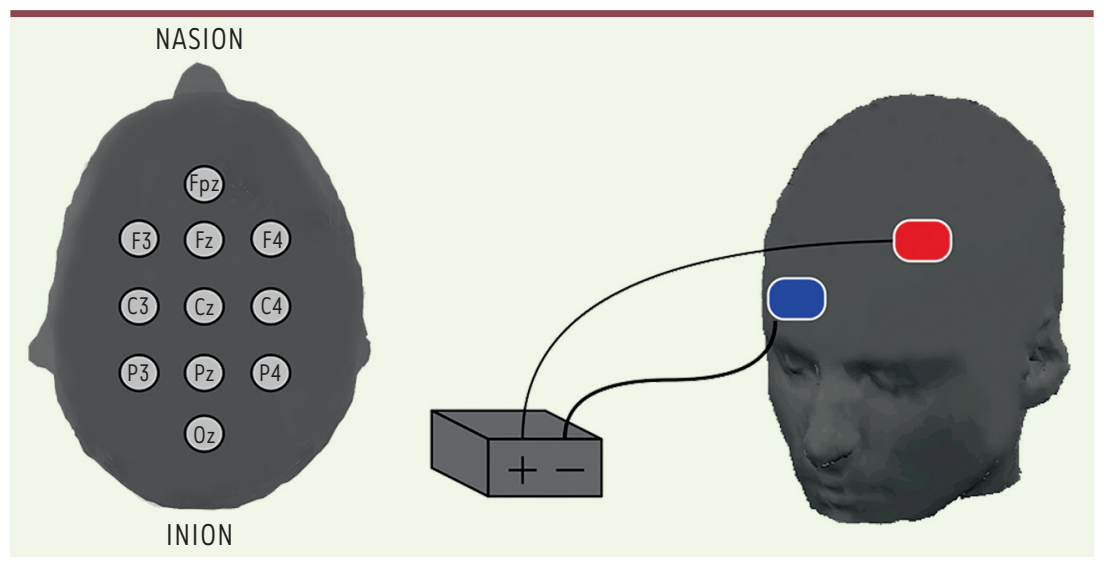

Figure 1. Illustration schématique de la technique de stimulation transcrânienne à courant continu. L'électrode rouge représente l'anode (chargée positivement) et l'électrode bleue représente la cathode (chargée négativement). Dans la partie gauche, le schéma représente les sites les plus courants de placement des électrodes selon le système $10-20$ relatif à I'électroencéphalographie. Le nasion est un repère anthropométrique situé au point de rencontre de la ligne médiane du nez et de la suture unissant les deux os propres du nez à l'os frontal. L'inion est le point le plus prominent de l'os occipital, dans la partie inférieure de l'arrière du crâne. Dans la partie droite, se situe un montage destiné à stimuler uniquement le cortex préfrontal gauche, avec l'anode placée sur le cortex préfrontal gauche et la cathode sur l'orbite contra-latérale. Ce montage constitue l'un des plus fréquents décrits dans la littérature sur l'utilisation de la tDCS (transcranial direct current stimulation) en psychiatrie.

\begin{tabular}{|c|c|c|c|c|c|}
\hline Technique & Intensité & Focalité & Coût & Invasivité & $\begin{array}{l}\text { Effets } \\
\text { indésirables }\end{array}$ \\
\hline tDCS & Faible & Modérée & Faible & Non & Faibles \\
\hline Électroconvulsivothérapie & Très élevée & Très faible & Élevé & Non & Élevés \\
\hline $\begin{array}{l}\text { Stimulation magnétique } \\
\text { transcrânienne }\end{array}$ & Faible & Élevée & Modéré & Non & Faibles \\
\hline Stimulation cérébrale profonde & Élevée & Très élevée & Très élevé & $\begin{array}{l}\text { Oui (implants } \\
\text { chirurgicaux) }\end{array}$ & Élevés \\
\hline
\end{tabular}

Tableau I. Comparaison de la technique de stimulation transcrânienne à courant continu (tDCS) avec les autres méthodes de neuromodulation utilisées en psychiatrie. Bien qu'une description exhaustive des autres méthodes de neuromodulation dépasse largement le cadre de cette synthèse, ces méthodes peuvent être résumées comme suit. L'électroconvulsivothérapie (aussi connue sous le nom de traitement par électrochocs) consiste en l'application de stimulations électriques (avec une intensité pouvant aller de 200 à 1600 milliampères) sur le scalp durant une à six secondes (le temps d'atteindre le seuil convulsif qui varie d'un individu à l'autre). Réalisée sous anesthésie générale, l'objectif de cette technique est de déclencher une crise d'épilepsie de type grand mal. La stimulation magnétique transcrânienne consiste en l'application d'impulsions magnétiques sur le cortex de façon indolore au moyen d’une bobine (solénoïde). La variation rapide de flux magnétique induit un champ électrique qui module l'activité des neurones situés dans le champ magnétique. À partir d'un certain seuil d'intensité, la modification rapide du champ magnétique induit localement une dépolarisation neuronale. La stimulation cérébrale profonde consiste à implanter par voie chirurgicale des électrodes, reliées par voie sous-cutanée à un stimulateur disposé en région sous-claviculaire, de sorte à délivrer une stimulation électrique à haute fréquence (par exemple, $130 \mathrm{~Hz}$ ) dans certaines structures cérébrales spécifiques.

\section{Prémisses}

L'utilisation de stimulation à courant continu pour soulager les troubles mentaux n'est pas récente. Vers 47, Scribonius Largus, le médecin de l'empereur romain Claude, narrait déjà avec grand soin comment placer efficacement une raie marbrée électrique (Torpedo marmorata ou torpille) sur le scalp afin de provoquer une décharge électrique importante et ainsi soulager des patients migraineux ou souffrant de douleurs [4]. De l'Antiquité à la Renaissance, des recommandations analogues sont fréquemment dépeintes dans les traités médicaux [4]. À la fin du XVIII ${ }^{\mathrm{e}}$ siècle, avec la découverte de la pile électrique, Galvani et Volta sont toutefois les premiers à expérimenter les corollaires physiologiques directs d'une stimulation électrique sur l'homme et l'animal [4]. Ces travaux ont le mérite d'avoir initié la recherche sur les effets cliniques de la stimulation par courant continu, et ce notamment par les travaux pionniers d'Aldini (le cousin de Galvani) [5] et d'Arndt [6] qui, au début du XIX siècle, traitaient déjà avec succès des cas sévères de dépression par cette méthode. Malgré des débuts enthousiastes, la découverte de l'électroconvulsivothérapie ${ }^{1}$ dans les

${ }^{1}$ L'électroconvulsivothérapie ou ECT est l'aboutissement des progrès scientifiques et techniques de l'ancienne méthode appelée « électrochocs ». Réalisée aujourd'hui sous anesthésie générale, l'ECT permet l'amélioration rapide de l'état de santé de certains patients par le recours à l'équivalent d'une crise convulsive, artificiellement provoquée en utilisant un courant faible et très bref appliqué à la surface du crâne. 
années 1930 [4] marque une perte d'intérêt pour la stimulation par courant continu. Dans les années 1960, un bref regain d'enthousiasme a toutefois lieu grâce aux recherches d'Albert [7] qui démontraient qu'une stimulation transcrânienne anodique, chez le rat, améliorait la consolidation en mémoire, alors qu'une stimulation cathodique réduisait cet effet. Néanmoins, ce regain d'intérêt est demeuré temporaire et limité à la recherche fondamentale. L'image négative associée à l'utilisation de l'électroconvulsivothérapie en psychiatrie ainsi que le développement de la pharmacothérapie et des interventions psychologiques, qui ont toutes deux radicalement transformé la prise en charge et le pronostic des patients, ont sans aucun doute contribué à ce désintérêt progressif.

Cela étant, les nouvelles molécules développées ces dernières années n'ont pas permis de progressions notables en termes d'efficacité, même si elles ont amélioré la tolérance aux traitements [8]. De même, bien que l'efficacité des procédures psychothérapeutiques soit de plus en plus éprouvée empiriquement, de nombreux patients ne répondent pas à ces interventions [8]. En outre, le développement récent de la tDCS dans d'autres secteurs cliniques ainsi que l'amélioration de la compréhension des mécanismes cérébraux mis en œuvre dans les troubles psychiatriques, et ce grâce aux progrès soutenus des neurosciences, ont constitué un terrain favorable à la réintégration progressive au cours de ces dernières années de la neuromodulation dans le domaine de la psychiatrie $[2,9](\rightarrow)$.

\section{Modus operandi}

$(\rightarrow)$ Voir la Nouvelle de B. Aouizerate et al., $m / s n^{\circ} 10$, octobre 2005, page 548

Avant de procéder à la stimulation, les zones où seront placées les électrodes doivent être préalablement désinfectées et exfoliées afin d'éliminer le plus d'obstacles entre le scalp et l'électrode. Les sites de stimulation sont habituellement déterminés à l'aide du système international $10-20^{2}$ relatif à l'électroencéphalographie. Le placement des électrodes est donc réalisé en fonction des régions dont on souhaite influencer le fonctionnement. La taille des électrodes varie généralement de $25 \mathrm{~cm}^{2}$ à $100 \mathrm{~cm}^{2}$ en fonction du niveau de précision souhaité, celles de plus petite taille permettant une stimulation plus focalisée. Les électrodes peuvent aussi être placées de manière plus précise en utilisant l'imagerie cérébrale anatomique.

Au début de chaque séance, les deux électrodes, entourées chacune d'un manchon en tissu spongieux, sont imbibées de sérum physiologique avant d'être placées sur le cuir chevelu du patient. Cette procédure permet d'assurer une conduction sécuritaire et de minimiser les éventuels effets désagréables relatifs au passage du courant à travers le scalp. Les électrodes sont habituellement maintenues en place à l'aide d'une bande de caoutchouc. La stimulation est généralement appliquée pour une durée de 10 à 30 minutes avec une intensité électrique variant de 1 à 2 milliampères $(\mathrm{mA})$ en fonction des études.

\footnotetext{
${ }^{2}$ Système international définissant le placement des électrodes.
}

\section{Mécanismes d'action}

La tDCS est une méthode de stimulation cérébrale superficielle non invasive qui permet de moduler l'excitabilité neuronale au moyen d'un faible champ électrique délivré sur le scalp par l'intermédiaire d'électrodes de polarité différente. Le courant circule de l'anode (chargée positivement) vers la cathode (chargée négativement) et seule une petite partie du courant délivré atteint le tissu cérébral (entre 40 et $60 \%$ ), le reste étant perdu au niveau du scalp et du crâne [10]. Cet effet est d'autant plus marqué que la distance entre les électrodes est importante [11]. Bien que le seuil de dépolarisation neuronale ne soit pas dépassé lors de I'application de la tDCS et qu'aucun potentiel d'action ne soit déclenché par la stimulation en tant que telle, les effets instantanés de cette stimulation sont visibles à deux niveaux. On observe en effet, d'une part, un décalage des potentiels de repos de la membrane vers un seuil de dépolarisation inférieur (une hyperexcitabilité), pour les neurones corticaux en regard de l'anode et, d'autre part, une augmentation du potentiel de repos de la membrane, avec un décalage du seuil de dépolarisation vers l'hyperpolarisation, pour les neurones corticaux en regard de la cathode.

Les effets post-stimulation sont, quant à eux, liés à une modulation des récepteurs $\mathrm{N}$-méthyl-D-aspartate (NMDA), récepteurs activés par le glutamate [12]. $\varepsilon n$ effet, il a été observé qu'un blocage des canaux ioniques voltage-dépendants, et plus particulièrement des canaux calciques, est associé à la suppression tant des effets immédiats que des effets post-stimulation [12]. A contrario, le blocage des récepteurs NMDA a pour conséquence la suppression des effets post-stimulation et le maintien des effets immédiats [13]. Dans l'ensemble, ces résultats indiquent que la modification du niveau de polarisation membranaire est responsable des effets immédiats de la tDCS, alors que la modulation des récepteurs NMDA sous-tendrait les effets post-stimulation. Les récepteurs NDMA sont impliqués dans les phénomènes de potentialisation à long terme (PLT) et de dépression à long terme (DLT), deux phénomènes de neuroplasticité bien documentés $[14](\rightarrow)$.

La PLT est un processus de renforcement synaptique qui correspond, à l'inverse de la DLT, à une aug- $(\rightarrow)$ Voir la Dernière heure de $D$. Choquet et B. Lounis, $m / s n^{\circ} 5$, mai 2008, page 548 mentation d'amplitude de la réponse post-synaptique à la suite d'une intense activation pré-synaptique. Cette activation conduit à une dépolarisation postsynaptique suffisante pour permettre une entrée massive de calcium via l'évacuation des ions magnésium 


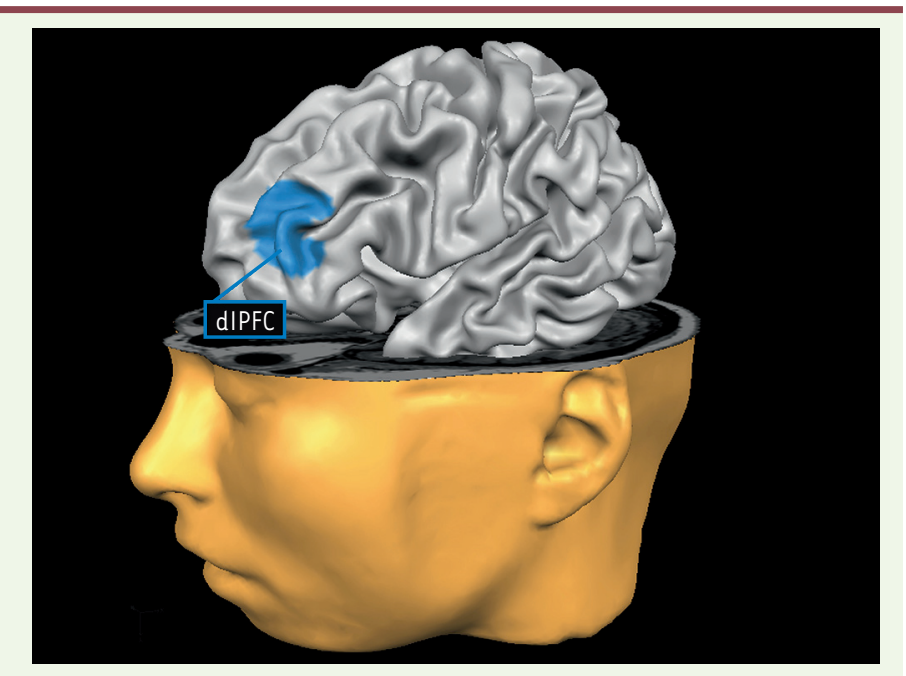

Figure 2. Illustration de la partie dorsolatérale du cortex préfontal. En raison de nombreuses publications indiquant la présence d'une hypoactivation de la partie dorsolatérale du cortex préfrontal (dorsolateral prefrontal cortex : dIPFC) dans l'hémisphère gauche chez les personnes souffrant de troubles dépressifs [18], cette région a longtemps constitué une cible privilégiée pour la stimulation anodique dans le traitement de la dépression par stimulation transcrânienne à courant continu. Au vu des résultats prometteurs de l'application de la technique dans le traitement de la dépression, cette région a également constitué, par la suite, une région d'intérêt dans les travaux ultérieurs sur l'application de cette méthode de stimulation auprès de populations présentant d'autres pathologies psychiatriques.

qui bloquent les récepteurs NMDA. Plus récemment, la participation de mécanismes de nature non synaptique, tels que des changements du niveau de $\mathrm{pH}$ membranaire ainsi que de production de protéines transmembranaires, a également été évoquée pour expliquer les effets post-stimulation [15]. Ainsi, bien que les effets instantanés semblent résulter d'une modification du potentiel membranaire, les mécanismes d'action sous-tendant les effets post-stimulation restent encore sujets à de nombreux débats.

\section{Applications cliniques en psychiatrie}

Bien qu'aucune méta-analyse n'ait encore été réalisée concernant l'efficacité de la tDCS sur le niveau de symptomatologie psychiatrique générale, des revues systématiques sont toutefois disponibles [16, 17]. À ce jour, les résultats de la recherche, relatifs à l'application de la tDCS dans la réduction du niveau de symptomatologie psychiatrique, peuvent être regroupés en quatre catégories distinctes : les troubles de l'humeur, les troubles liés aux addictions, les troubles psychotiques et les troubles anxieux. Ci-après, nous illustrerons brièvement ces travaux par la description d'essais cliniques contrôlés et randomisés en double-aveugle qui ont été menés pour chacun de ces troubles ${ }^{3}$.

${ }^{3}$ Nous invitons cependant le lecteur intéressé par une description exhaustive de la littérature existante à consulter les revues systématiques mentionnées en références $[16,17]$.
Des travaux ayant indiqué l'existence d'une hypoactivation de la partie dorsolatérale du cortex préfrontal gauche (dorsolateral prefrontal cortex-dIPFC) (Figure 2) chez les patients présentant un trouble dépressif [18], des études ont été menées afin d'examiner les effets cliniques de l'application d'une stimulation anodique de 20 minutes sur cette région (avec la cathode localisée sur l'orbite controlatérale - c'est-à-dire du côté opposé à l'anode). En comparaison à une stimulation placébo (une procédure similaire mais incluant l'arrêt de la stimulation après quelques secondes), une amélioration de la symptomatologie de ces patients, avec un maintien des bénéfices jusqu'à 1 mois après la fin de l'intervention en l'absence de tout autre traitement, a été rapportée $[19,20]$. Dans la plupart de ces études, l'intensité était de $2 \mathrm{~mA}$ et les séances de stimulation étaient administrées sur des durées allant de 5 à 15 jours consécutifs. Plus récemment, la supériorité, en termes de réduction du niveau de symptomatologie, d'une procédure de stimulation bifrontale (I'anode placée sur le dIPFC gauche et la cathode sur le dIPFC droit ${ }^{4}$ ) administrée durant 10 jours consécutifs à raison de 30 minutes par jour avec une intensité de $2 \mathrm{~mA}$, a également été observée, en comparaison à une procédure de stimulation placebo [2], 22]. En outre, le bénéfice de l'administration d'une telle stimulation bifrontale en association avec un traitement à la sertraline (un antidépresseur de type inhibiteur sélectif de la recapture de la sérotonine), a récemment été démontré, en comparaison à l'administration isolée de chacun de ces ingrédients thérapeutiques [21]. Cela étant, à l'exception du trouble dépressif majeur, peu d'études ont examiné l'effet de la tDCS pour les troubles de l'humeur. À la suite de l'observation d'une hypoactivation du dIPFC droit chez les patients souffrant de troubles bipolaires, un effet bénéfique d'une stimulation anodique sur cette région a toutefois été décrit chez ces patients [23].

En ce qui concerne les addictions, quelques études impliquant un montage bifrontal similaire à celui décrit dans les troubles dépressifs ont été réalisées. Elles indiquent une réduction transitoire du niveau de symptomatologie après l'application d'une séance de tDCS (2 $\mathrm{mA}$ ) auprès de patients alcoolo-dépendants [24] et aussi tabaco-dépendants [25]. L'effet bénéfique sur le niveau de consommation de patients tabacodépendants d'un tel montage avec une intensité de $2 \mathrm{~mA}$, administré durant 5 jours consécutifs à raison de 25 minutes de stimulation par jour, a également été rapporté [26]. ${ }^{4}$ Respectivement, $\mathrm{F} 3$ et $\mathrm{F} 4$ selon le système international $10-20$ relatif à
l'électroencéphalographie (Figure 1 ). 
Plus récemment, des travaux ont été menés auprès de patients souffrant de troubles psychotiques. En vue de réduire la fréquence des hallucinations auditives, des études visant une stimulation cathodique du cortex temporo-pariétal gauche ${ }^{5}$ (supposé jouer un rôle dans ces hallucinations auditives), ont été réalisées à raison de 2 sessions ( $2 \mathrm{~mA}$ ) de 20 minutes par jour durant 5 jours consécutifs. Dans ce cas, la tDCS est associée à une réduction des hallucinations auditives ainsi qu'à une amélioration générale de la symptomatologie, en comparaison à une stimulation placebo $[27,28]$. Des études récentes suggèrent également un effet bénéfique d'une stimulation par montage bifrontal sur les symptômes négatifs de patients souffrant de troubles psychotiques [16].

Enfin, bien que les troubles anxieux constituent la catégorie de troubles psychiatriques ayant la prévalence la plus élevée, peu d'études ont évalué l'efficacité de la tDCS au sein de ces troubles. À ce jour, seule une étude de cas d'un patient souffrant d'un trouble obsessionnel-compulsif a été réalisée. Elle montre les effets bénéfiques sur le niveau de symptomatologie d'une séance de 20 minutes de tDCS à 2 mA, avec la cathode placée sur le dIPFC gauche et l'anode sur l'épaule du patient [29].

\section{Perspectives et conclusion}

Malgré les résultats prometteurs des études que nous avons évoquées, les effets thérapeutiques de la tDCS auprès des populations souffrant de troubles psychiatriques demeurent encore aujourd'hui le sujet de nombreuses incertitudes. Ainsi, bien que la majorité des protocoles ayant démontré une efficacité clinique aient utilisé des sessions de stimulation de $2 \mathrm{~mA}$ sur des durées allant de 20 à 30 minutes, le nombre de sessions, l'intervalle entre les séances, ou encore le type de montage varient considérablement selon les études [16]. L'efficacité clinique de la tDCS demeure également inexplorée pour de nombreux troubles, en particulier pour les troubles anxieux (par exemple, le trouble anxieux généralisé). À l'avenir, les travaux devront donc impérativement préciser la nature exacte des troubles pour lesquels la tDCS est susceptible de constituer un outil thérapeutique. Ces travaux devront également spécifier les paramètres requis afin d'optimiser l'efficacité clinique de cette technique. Au regard des travaux réalisés chez l'animal et le sujet sain [30], l'influence, sur la réponse à la tDCS, de facteurs comme l'apparition de comorbidité psychiatrique ou les éventuelles prises conjointes de pharmacothérapie devra être évaluée. La qualité méthodologique des essais cliniques reste faible [16], plus particulièrement en raison de l'absence d'étude multicentrique intégrant de larges échantillons permettant la comparaison de la tDCS avec les traitements pharmacologiques et psychothérapeutiques actuellement en vigueur, une étape nécessaire avant toute implémentation potentielle de cette technique au sein de services de soins de santé mentale.

Au-delà de l'application isolée de la tDCS afin de réduire le niveau de symptomatologie de certains patients, de nombreux chercheurs se tournent depuis peu vers l'exploration des effets de la tDCS sur l'effi-

$\overline{{ }^{5} \text { Respectivement entre } \mathrm{P} 3 \text { et } \mathrm{T} 3 \text { selon le système international 10-20 relatif à l'électroencéphalographie. }}$ cience de processus cognitifs (par exemple l'attention ou la mémoire) [31]. De façon générale, la tDCS est administrée, dans ces études récentes, de manière concomitante à l'exécution d'une tâche informatisée qui évalue le ou les processus cognitifs analysés [31]. Récemment, des travaux ont démontré la possibilité d'améliorer significativement la récupération de capacités cognitives après un accident vasculaire cérébral par l'utilisation de séances de remédiation cognitive couplées à une procédure de tDCS [32]. Ces résultats ont stimulé l'envie d'évaluer une telle démarche pour son application dans la remédiation de processus cognitifs jouant un rôle psychopathogène qui a été avéré en psychopathologie [33-36]. Bien que ces travaux soient encore aujourd'hui à un stade quasiembryonnaire, les premiers résultats s'avèrent particulièrement prometteurs [37-39]. Étant donné le faible coût de la tDCS, celle-ci pourrait rapidement renouveler l'exercice de l'intervention sur ces processus psychopathogènes [39]. Enfin, au-delà des implications cliniques possibles de la tDCS, la possibilité de manipuler expérimentalement l'excitabilité neuronale de régions cibles et d'en examiner les effets directs sur l'efficience de processus cognitifs psychopathogènes, devrait permettre, à terme, de dépasser les écueils rencontrés dans les recherches réalisées auparavant, comme l'utilisation de plans de recherche qui ne permettent pas l'inférence de relations causales [37-40]. $\diamond$

\section{SUMMARY}

Transcranial direct current stimulation: new clinical roadmaps for psychiatric research

Transcranial direct current stimulation (tDCS) is a neuromodulatory technique that has undergone intensive research over the past decade with promising results. tDCS is based on the application of weak, direct current over the scalp, leading to cortical hypo- or hyperpolarization according to the specified parameters. Recent studies have shown that tDCS is able to induce potent changes in cortical excitability as well as to elicit longlasting modifications in brain activity. Over the last decade, tDCS physiological mechanisms of action have been intensively investigated. This research has given support for the investigation of tDCS applications in a wide range of clinical populations, including patients with post-stroke motor and language deficits, chronic pain, and tinnitus. Recently, its efficacy to treat psychiatric conditions has been explored increasingly. In this review, we will gather clinical studies involving tDCS to ameliorate psychiatric symptoms and discuss reasonable next steps in this direction. $\diamond$ 


\section{REMERCIEMENTS}

Cette recherche a reçu le soutien d'une bourse (FC78142) de recherche postdoctorale du Fonds national de la recherche scientifique belge (FRS-FNRS) attribué à Alexandre Heeren. La rédaction de cet article a également reçu le soutien de la Fondation belge pour la vocation («Vocatio ») et de la Bourse d'excellence scientifique de la Fédération WallonieBruxelles-International - Pôle de compétitivité du Plan Marshall 2.Vert en sciences du vivant - BIOWIN (Belgique), toutes deux attribuées à Alexandre Heeren, ainsi que d'une bourse de la Belgian American Educational Foundation, attribuée à Elisabeth Colon.

\section{LIENS D'INTÉRÊT}

Les auteurs déclarent n'avoir aucun lien d'intérêt concernant les données publiées dans cet article.

\section{RÉFÉRENCES}

1. Brunoni AR, Amadera J, Berbel B, et al. A systematic review on reporting and assessment of adverse effects associated with transcranial direct current stimulation. Int J Neuropsychopharmacol $2011 ; 14: 1133-45$.

2. Kuo MF, Paulus $W$, Nitsche MA. Therapeutic effects of non-invasive brain stimulation with direct currents (tDCS) in neuropsychiatric diseases. Neuroimage $2014 ; 85$ : 948-60.

3. Chevreul K, Prigent A, Bourmaud A, et al. The cost of mental disorders in France. Eur Neuropsychopharmacol 2013;23:879-86.

4. Kellaway P. The part played by the electric fish in the early history of bioelectricity and electrotherapy. Bull Hist Med $1946 ; 20$ : 112-37.

5. Aldini G. Essai théorique et expérimental sur le galvanisme. Réédition. Paris : Gallica, 1804.

6. Arndt R. Die electricitat in der psychiatrie. Arch Psychiatr Nervenkr $1869 ; 2: 259-61$.

7. Albert DJ. The effects of polarizing currents on the consolidation of learning. Neuropsychologia $1966 ; 4: 65-77$.

8. Mojtabai R, Olfson M, Sampson, NA, et al. Barriers to mental health treatment: results from the National comorbidity survey replication (NCS-R). Psychol Med 2011 ; 41 : 1751-61.

9. Aouizerate B, Martin-Guehl C, Cuny $\varepsilon$, et al. Stimulation cérébrale profonde du striatum ventral dans le traitement du trouble obsessionnel-compulsif avec dépression majeure. Med Sci (Paris) $2005 ; 21: 811-3$

10. Miranda P, Lomarev M, Hallett M. Modeling the current distribution during transcranial direct current stimulation. Clin Neurophysiol 2006 ; 117 : 1623-9.

11. Moliadze V, Antal A, Paulus W. Electrode-distance dependent after-effects of transcranial direct and random noise stimulation with extracephalic reference electrodes. Clin Neurophysiol 2010 ; $121: 2165-71$.

12. Nitsche $M$, Fricke $K$, Henschke U, et al. Pharmacological modulation of cortical excitability shifts induced by transcranial direct current stimulation in humans. J Physiol $2003 ; 553: 293-301$.

13. Nitsche M, Jaussi W, Liebetanz D, et al. Consolidation of externally induced human motor cortical neuroplasticity by D-cycloserine. Neuropsychopharmacology 2004 ; 29 : 1573-8.

14. Choquet $D$, Lounis $B$. Mobilité des récepteurs du glutamate : Un nouveau mécanisme de contrôle de la transmission synaptique rapide dans le cerveau. Med Sci (Paris) 2008 ; $24: 548-50$

15. Ardolino G, Bossi B, Barbieri S, et al. Non-synaptic mechanisms underlie the after-effects of cathodal transcutaneous direct current stimulation of the human brain.J Physiol $2005 ; 568: 653-63$

16. Kekic M, Boysen $\varepsilon$, Campbell IC, Schmidt U. A systematic review of the clinical efficacy of transcranial direct current stimulation (tDCS) in psychiatric disorders. J Psychiatr Res 2016; 74: 70-86.

17. Kuo MF, Paulus $W$, Nitsche. Therapeutic effects of non-invasive brain stimulation with direct currents (tDCS) in neuropsychiatric diseases. Neuroimage $2014 ; 85$ : 948-60.

18. Fitzgerald PB, Oxley T), Laird, AR, et al. An analysis of functional neuroimaging studies of dorsolateral prefrontal cortical activity in depression. Psychiatry Res 2006 ; 148 : 33-45.

19. Boggio PS, Rigonatti SP, Ribeiro RB, et al. A randomized, double-blind clinical trial on the efficacy of cortical direct current stimulation for the treatment of major depression. Int J Neuropsychopharmacol $2008 ; 11: 249-54$

20. Loo CK, Alonzo A, Martin D, et al. Transcranial direct current stimulation for depression: 3-week, randomized, sham-controlled trial. BrJ Psychiatry $2012 ; 200: 52-9$.

21. Brunoni AR, Valiengo $L$, Baccaro $A$, et al. The sertraline vs electrical current therapy for treating depression clinical study: results from a factorial, randomized, controlled trial. JAMA Psychiatry $2013 ; 70: 383-91$
22. Dell'Osso B, Zanoni S, Ferrucci R, et al. Transcranial direct current stimulation for the outpatient treatment of poor-responder depressed patients. Eur Psychiatry $2012 ; 27: 513-7$.

23. Schestatsky P, Janovik N, Lobato MI, et al. Rapid therapeutic response to anodal tDCS of right dorsolateral prefrontal cortex in acute mania. Brain Stimul $2013 ; 6$ : 701-11

24. Boggio PS, Sultani N, Fecteau S, et al. Prefrontal cortex modulation using transcranial DC stimulation reduces alcohol craving: a double-blind, shamcontrolled study. Drug Alcohol Depend $2008 ; 92: 55-60$

25. Fregni F, Liguori P, Fecteau $S$, et al. Cortical stimulation of the prefrontal cortex with transcranial direct current stimulation reduces cue-provoked smoking craving: a randomized, sham-controlled study. J Clin Psychiatry $2008 ; 69: 32-40$.

26. Boggio PS, Liguori P, Sultani N, et al. Cumulative priming effects of cortical stimulation on smoking cue-induced craving. Neurosci Lett 2009; 463 : 82-6.

27. Brunelin J, Mondino M, Gassab L, et al. Examining transcranial direct-current stimulation (tDCS) as a treatment for hallucinations in schizophrenia. Am J Psychiatry $2012 ; 169$ : 719-24.

28. Homan P, Kindler J, Federspiel A, et al. Muting the voice: a case of arterial spins labeling-monitored transcranial direct current stimulation treatment of auditory verbal hallucinations. Am J Psychiatry $2011 ; 168$ : 853-4.

29. Volpato C, Piccione F, Cavinato M, et al. Modulation of affective symptoms and resting state activity by brain stimulation in a treatment-resistant case of obsessive-compulsive disorder. Neurocase $2013 ; 19: 360-70$.

30. Li LM, Uehara K, Hanakawa T. The contribution of interindividual factors to variability of response in transcranial direct current stimulation studies. Front Cell Neurosci $2015 ; 9: 181$.

31. Filmer HL, Dux PE, Mattingley JB. Applications of transcranial direct current stimulation for understanding brain function. Trends Neurosci 2014 ; 37 : 742-53.

32. Lefebvre $S$, Dricot $L$, Laloux $P$, et al. Neural substrates underlying stimulation-enhanced motor skill learning after stroke. Brain $2015 ; 138$ 149-63.

33. Maurage P, de Timary P, Billieux J, et al. Attentional alterations in alcohol dependence are underpinned by specific executive control deficits. Alcohol Clin Exp Res 2014 ; 38 : 2105-12.

34. Coussement $C$, Heeren $A$. Vers une architecture cognitive du maintien du biais attentionnel envers la menace dans l'anxiété : une approche par comparaison de modèles. L'Année Psychologique 2015 ; 115 : 665-90.

35. Heeren A, Maurage P, Perrot $\mathrm{H}$, et al. Tinnitus specifically alters the topdown executive sub-component of attention: evidence from the Attention network task. Behav Brain Res $2014 ; 269$ : 147-54

36. Heeren A, Mogoase C, Philippot P, et al. Attention bias modification for social anxiety: A systematic review and meta-analysis. Clin Psychol Rev $2015 ; 40: 76-90$.

37. Heeren A, Baeken C, Vanderhasselt MA, et al. Impact of anodal and cathoda transcranial direct current stimulation over the left dorsolateral prefrontal cortex during attention bias modification: An eye-tracking study. PLoS One $2015 ; 10$ : e0124182

38. Clarke PJ, Browning M, Hammond G, et al. The causal role of the dorsolateral prefrontal cortex in the modification of attentional bias: evidence from transcranial direct current stimulation. Biol Psychiatry 2014 ; 76 : 946-52.

39. De Raedt R, Vanderhasselt MA, Baeken C. Neurostimulation as an intervention for treatment resistant depression: From research on mechanisms towards targeted neurocognitive strategies. Clin Psychol Rev $2015 ; 41: 61-9$

40. Maurage $P$, Heeren A, Pesenti M. Does chocolate consumption really boost Nobel Award chances? The peril of over-interpreting correlations in health studies. J Nutr 2013; 143: 931-3.

\section{TIRÉS À PART}

A. Heeren

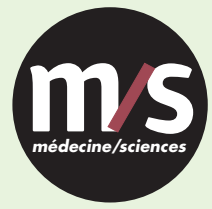

Tarifs d'abonnement $\mathrm{m} / \mathrm{s}-2016$

Abonnez-vous

à médecine/sciences
$>$ Grâce à $m / s$, vivez en direct les progrès des sciences biologiques et médicales

Bulletin d'abonnement page 724 dans ce numéro de $\mathrm{m} / \mathrm{s}$

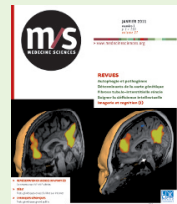

